\title{
ORIGINAL
}

\section{HEPATITIS B Y C EN PERSONAS CON VIH DEL INSTITUTO PARA EL DESARROLLO HUMANO}

\author{
HEPATITIS B AND C IN PEOPLE WITH HIV \\ FROM THE INSTITUTE FOR HUMAN DEVELOPMENT
}

\author{
Dr. Jhemel Garay Quiroga', Dr. Edgar Valdez Carrizo²
}

${ }^{1}$ Especialista en Medicina Tropical, Responsable del Servicio de Salud del Instituto para el Desarrollo Humano

2 Magíster en Salud Pública, Director del Instituto para el Desarrollo Humano.

Correspondencia a:

Nombre: Dr. Jhemel Garay Quiroga

Correo: jhemelgaray@gmail. com

ORCID: https://orcid.org/00000002-4948

Telf. y celular: $+(591) 77781143$

Palabras clave: VIH, Hepatitis B, Hepatitis C, Tenofovir, Lamivudina.

Keywords: HIV, hepatitis B, hepatitis $C$, tenofovir, lamivudina.

Procedencia y arbitraje: no comisionado, sometido a arbitraje externo.

Recibido para publicación: 12 febrero del 2020 Aceptado para publicación: 12 febrero del 2020

Citar como:

Garay Quiroga J, Valdez Carrizo E. Hepatitis $B$ y $C$ en personas con VIH del instituto para el desarrollo humano. Rev Cient Cienc Med 2021;24(1): 122 - 126

\section{RESUMEN}

Introducción: La coinfección del VIH con la hepatitis B o C es causa de patología hepática crónica, con diversas seroprevalencias en diferentes regiones del mundo. Objetivo: Conocer la seroprevalencia de hepatitis $B$ y $C$ en personas con VIH que acuden a consulta en el Instituto para el Desarrollo Humano, Cochabamba-Bolivia, gestión 2017-2018. Materiales y Métodos: Estudio descriptivo, prospectivo-transversal, cuantitativo, no experimental. Se analizaron los resultados serológicos de los marcadores:Antígeno de superficie del virus hepatitis $B$, anticuerpo anti-core del virus de hepatitis $B$, anticuerpo contra el virus hepatitis $C$; historial de vacuna para hepatitis B y esquema de tratamiento antirretroviral, de 195 personas con $\mathrm{VIH}$ en el Instituto para el Desarrollo Humano, se realizó análisis bivariado para la obtención de datos. Resultados: La seroprevalencia obtenida para hepatitis $\mathrm{B}$ es de $7,7 \%$ y hepatitis $\mathrm{C}$ de $0,5 \%$; la coinfección entre $\mathrm{VIH}$ y hepatitis $\mathrm{B}$ es de $80 \%$ con esquema antirretroviral tenofovir/lamivudina. El grupo poblacional homosexual tiene un riesgo de 5,5 veces más de tener la co-infección de $\mathrm{VIH}$ y hepatitis $\mathrm{B}$ con un valor de $\mathrm{p}$ de 0,006 ; con relación a la inmunización para hepatitis $\mathrm{B}$ solo el $9,2 \%$ de los pacientes cuentan con el esquema completo. Conclusión: Es imperativo ofertar las pruebas para hepatitis $B$ y $\mathrm{C}$ a todas las personas con $\mathrm{VIH}$; haciendo énfasis en los grupos más vulnerables (homosexuales); es importante el abastecimiento de dosis para la inmunización completa contra la hepatitis B en todos los servicios de salud públicos del país.

\section{ABSTRACT}

Introduction: HIV co-infection with hepatitis B or C is the cause of chronic liver disease, with various seroprevalences in different regions of the world. Objective: To know the seroprevalence of hepatitis $B$ and $C$ in people with HIV who come for consultation at the Institute for Human Development, Cochabamba-Bolivia, management 2017-2018. Materials and methods: Descriptive, prospective-cross-sectional, quantitative, non-experimental study. The serological results of the markers were analyzed: Hepatitis B virus surface antigen, Hepatitis $B$ virus anti-core antibody, Hepatitis $C$ virus antibody; Hepatitis $B$ vaccine history and antiretroviral treatment scheme of 195 people with HIV at the Institute for Human Development, bivariate analysis was performed to obtain data. Results: The seroprevalence obtained for hepatitis B is $7.7 \%$ and hepatitis C $0.5 \%$; coinfection between HIV and hepatitis B is $80 \%$ with the antiretroviral regimen tenofovir / lamivudine. The homosexual population group has a 5.5 times risk of having HIV and hepatitis $B$ co-infection with a $P$ value of 0.006 ; Regarding immunization for hepatitis B, only $9.2 \%$ of patients have the complete scheme. Conclusion: It is imperative to offer hepatitis B and $\mathrm{C}$ testing to all people with HIV; emphasizing the most vulnerable groups (homosexuals); The provision of doses for complete immunization against hepatitis B in all public health services in the country is important.

\section{INTRODUCCIÓN}

a hepatitis $B$, hepatitis $\mathrm{C}$ y VIH, tienen en común las vías de trasmisión y factores epidemiológicos; la coinfección de las personas con VIH y hepatitis B o C, son causa frecuente de hepatopatías crónicas; se han realizado varios estudios en diferentes países, demostrando la prevalencia de coinfección entre $\mathrm{VIH}$ y hepatitis B de 4,8 al 15\%, coinfección de VIH y hepatitis $C$ de 0 al 42,5\%"; pero se debe considerar que la prevalencia de las mismas difiere de acuerdo a grupos de riesgo y diferentes regiones ${ }^{2}$. En personas coinfectadas con $\mathrm{VIH}$ y hepatitis $\mathrm{B}$, la carga viral es más elevada que en personas con una sola infección, por lo tanto tienen mayor riesgo de evolucionar a cirrosis en más corto tiempo ${ }^{3}$.

En América 2,8 millones de personas están infectadas de forma crónica por la hepatitis B (VHB), en la población general, la prevalencia del antígeno de superficie del VHB (VHBsAg) es 
de 0,28\%; para Bolivia esta seroprevalencia del VHBsAg es del 0,44\% ${ }^{4}$.

En comparación los grupos más vulnerables, presentan una mayor prevalencia de infección por el VHB. La seroprevalencia en trabajadoras sexuales del VHBsAg es del 1,5\%; hombres que tienen relaciones sexuales con otros hombres (HSH) de 2,9\%; personas privadas de libertad $8,6 \%$, personas que usan drogas parenterales de $3,3 \%$; y en usuarios de drogas no parenterales de $1,5 \%{ }^{4}$.

Con relación a la infección crónica de hepatitis $C$ en América, 7,2 millones de personas padecen esta patología, con prevalencia de 0,73\% en población general del continente americano, solo en Latinoamérica y el Caribe de $0,65 \%{ }^{4}$.

Las prevalencias más elevadas de hepatitis $C$ también se observan en los grupos más vulnerables; la seroprevalencia de anticuerpo contra VHC (Anti-VHC) en usuarios de drogas parenterales es de $49 \%$ y de drogas no parenterales es de $4 \%, \mathrm{HSH}$ del $3 \%$ y en trabajadoras sexuales del $2 \% 4$.

Actualmente gracias al Tratamiento Antirretroviral (TARV) se ha logrado prolongar la sobrevida de las personas con $\mathrm{VIH}$, pero a la vez, empezaron a presentar las complicaciones crónicas por coinfecciones de hepatitis B o C como la hepatopatía, causa de morbimortalidad frecuente ${ }^{2}$.

Los estudios realizados en Bolivia sobre el VIH, carecen de aspectos técnicos y responden mayormente a aspectos ideológicos, influyendo de esta manera en la calidad de los datos epidemiológicos ${ }^{5}$. Por tal motivo se realiza dicha investigación, con el objetivo de conocer la seroprevalencia de hepatitis $\mathrm{B}$ y $\mathrm{C}$ en personas con $\mathrm{VIH}$ que acuden a consulta en el Instituto para el Desarrollo Humano (IpDH), Cochabamba-Bolivia entre las gestiones 2017-2018.

\section{MATERIALES Y MÉTODOS}

El presente estudio es de tipo descriptivo, prospectivo-transversal, cuantitativo, no experimental.

Se analizaron los resultados serológicos de los marcadores: antígeno de superficie del VHB (VHBsAg), anticuerpo contra el core de VHB (Anti-VHBC) y anticuerpo contra VHC (Anti-VHC), de todas las personas con $\mathrm{VIH}$ que acuden regularmente para su control y seguimiento médico al Instituto para el Desarrollo Humano.
Se realizaron pruebas rápidas de hepatitis $B$ y $\mathrm{C}$ a todas las personas que no hayan realizado su laboratorio serológico; el resultado fue registrado en la historia clínica y en el registro institucional de pruebas rápidas. Se solicitó que todo paciente con prueba rápida de $\mathrm{VHBsAg}$ de resultado positivo se le realice también serología para Anti-VHBc.

Los pacientes con resultado positivo para VHBsAg (6 personas), Anti-VHBc (12 personas) y Anti-VHC (una persona), se les revisó el historial clínico para identificar el tipo de tratamiento antirretroviral utilizado y verificar que la mayoría tenga en su esquema tenofovir/lamivudina para tratar la co-infección de $\mathrm{VIH}$ y hepatitis $\mathrm{B}$.

Se obtuvo de las historias clínicas el grupo poblacional al que pertenecen las personas con VIH del Instituto para el Desarrollo Humano, coinfectados por hepatitis B o C; también todo paciente que haya realizado la inmunización completa (tres dosis) para hepatitis B que además cuenten con el carnet de vacunación correspondiente para identificar la prevalencia de personas con $\mathrm{VIH}$ inmunizadas para hepatitis $\mathrm{B}$.

El protocolo del estudio fue aprobado por el Comité de Bioética de la Facultad de Medicina de la Universidad Mayor de San Simón.

La población objeto del estudio es toda persona con $\mathrm{VIH}$, mayor de 18 años de edad que acude regularmente a control médico y con diagnóstico de coinfección por virus de la hepatitis $\mathrm{B}$ y/o C, comprobado por medio de examen serológico con resultado reactivo o positivo para VHBsAg, Anti-VHBc y Anti-VHC, con tratamiento antirretroviral con el esquema que contenga tenofovir y lamivudina; y con inmunización completa para hepatitis B. Entre otras variables tomadas en cuenta están la edad, sexo y orientación sexual.

La recolección de datos se realizó en las instalaciones del Instituto para el Desarrollo Humano.

El estudio se llevó a cabo en el Instituto para el Desarrollo Humano en el municipio de Cercado-Cochabamba-Bolivia en el periodo comprendido del año 2017 al 2018.

La muestra son los 195 pacientes registrados para control y seguimiento clínico por infección de VIH en el Instituto para el Desarrollo Humano hasta diciembre del 2018.

\section{Criterios de inclusión}

Toda persona con VIH mayor de 18 años del Instituto para el Desarrollo Humano que se rea- 
lice las pruebas serológicas para VHB y VHC entre el año 2017 y 2018 con un registro escrito en la historia clínica.

\section{Criterios de exclusión}

Toda persona con VIH mayor de 18 años del Instituto para el Desarrollo Humano que no realice las pruebas serológicas para VHB y VHC entre el año 2017 y 2018.

Toda persona con VIH menor de 18 años del Instituto para el Desarrollo Humano.

Los datos obtenidos fueron introducidos en el programa Epi Info versión 3.5.4 para su análisis e interpretación.

RESULTADOS

En el estudio fueron incluidas 195 personas con VIH, atendidas en el Instituto para el Desarrollo Humano, de las cuales $21 \%$ (n: 41 ) eran de sexo femenino y $79 \%$ ( $n$ : 154$)$ de sexo masculino, con una media de edad de 31,5 años. En general la seroprevalencia de coinfecciones son las siguientes: Para VHB es de 7,7\% (n: 15), y para VHC de $0,5 \%$ (n: 1 ). No se observaron coinfecciones múltiples por ambos virus en personas con $\mathrm{VIH}$.

Con relación a la coinfección de VIH con VHB, la seroprevalencia de VHBs Ag positivo es de $3,1 \%$ (n: 6) y de Anti-VHBc positivo de 6,2\% (n: 12) existiendo una asociación estadísticamente significativa $(p=0,0000001)$ y en relación a la seroprevalencia de Anti-VHC es de 0,5\% (n: 1) observándose también una asociación estadísticamente significativa ( $p=0,0000001)$.

De acuerdo al sexo, las prevalencias para la coinfección VIH/VHB fueron en mujeres $6,7 \%$ (n: 1) y varones $93,3 \%$ (n: 14), no existiendo una asociación estadísticamente significativa con un $\mathrm{OR}=0.25$ [IC 95\%=(0.01-1.48)]; para coinfección VIH/VHC mujeres $100 \%$ (n: 1), varones $0 \%$.

De las 15 personas coinfectadas con $\mathrm{VIH}$ y VHB, el $80 \%$ (n: 12 ) reciben Tenofovir con Lamivudina como parte de su esquema de tratamiento antirretroviral y el 20\% (n: 3) aún no cuenta con tratamiento antirretroviral. Se demostró que en los casos de coinfección VIH y VHB el recibir tenofovir con lamivudina dentro su esquema antirretroviral no hay relación significativa con un OR=0.93 [IC 95\%=(0.26-4.31)].

El grupo poblacional que reúne a la mayor cantidad de coinfectados con $\mathrm{VIH}$ y VHB es el grupo de los hombres que tienen sexo con hombres concentrando al 12\% (n: 13); en cambio en la población general esta coinfección es de 2\% (n: 2) (Ver Tabla 1); por tanto los $\mathrm{HSH}$ tiene un riesgo de 5,5 veces más de tener la co-infección de VIH y VHB con un valor de $\mathrm{p}$ de 0,006 , siendo esta relación estadísticamente significativa.

La coinfección VIH y VHC está presente en $1,2 \%$ ( $\mathrm{n}: 1$ ) en la población general y ninguno en los HSH (Ver Tabla 2), con un riesgo de 0, dando el valor de p de 0,21 sin significancia estadística.

Tabla 1. Co-infección VIH y hepatitis $B$ o $C$ según población

\begin{tabular}{|lll|}
\hline Coinfección VIH/hepatitis B & $n$ & Porcentaje \\
\hline $\begin{array}{l}\text { Hombres que tiene relaciones sexuales con } \\
\text { otros hombres }\end{array}$ & $13 / 110$ & $12 \%$ \\
\hline Población general & & \\
\hline
\end{tabular}

\begin{tabular}{lll}
\hline Coinfección VIH/hepatitis C & $\mathrm{n}$ & Porcentaje \\
\hline $\begin{array}{l}\text { Hombres que tiene relaciones sexuales con } \\
\text { otros hombres }\end{array}$ & $0 / 110$ & $0 \%$ \\
\hline Población general & & \\
\hline
\end{tabular}

Con relación a la inmunización para $\mathrm{VHB}$, de las 195 personas con VIH atendidas en el IpDH, solo se encuentran inmunizadas el 9,2\% (n: 18), ninguna de las personas reactivas para VHB fueron inmunizadas, se observó que esta relación no es estadísticamente significativa con un $\mathrm{OR}=0$ [IC 95\%=(0.0-2.16)] y valor de $\mathrm{p}=0,22$.

\section{DISCUSIÓN}

Nos permite observar la coinfección de la hepatitis viral por VHB y VHC en una población con VIH.

Las seroprevalencias de VHB y VHC en personas con $\mathrm{VIH}$, son inferiores a otras investigaciones similares; la seroprevalencia para VHB fue de $7,7 \%$, menor a la encontrada en Argentina $68,8 \%$ y $17 \%^{2}$, Cuba $14,4 \%^{6}$ y Venezuela $15 \%{ }^{7}$; pero mayor a la encontrada en España 5,4\% .

En cuanto a la seroprevalencia de VHC de $0,5 \%$, también fue mucho menor en comparación con otros estudios en Argentina 88.3\% y $32 \%{ }^{2}$, Cuba $20 \%{ }^{6}$ y Venezuela $5 \%{ }^{7}$.

En la co-infección de VIH con VHB, la seroprevalencia de los diferentes marcadores también fue inferior a series encontradas en otros estudios; el VHBs Ag positivo en el estudio fue de $3,1 \%$ y de Anti-VHBc positivo de 6,2\%; a comparación de Argentina con 14,5\% y 58,5\%; Brasil $10,3 \%$ y $38,7 \%$ respectivamente ${ }^{1,2}$. 
La seroprevalencia de la coinfección $\mathrm{VIH} / \mathrm{VHB}$ es mayor en varones $93,3 \%$ que en mujeres $6,7 \%$, similar a los estudios realizados en España donde los hombres tenían la coinfección en un $90,7 \%$ y las mujeres $9,3 \%{ }^{8}$; Paraguay con $89 \%$ varones y $11 \%$ mujeres ${ }^{9}$.

En el presente estudio, el único caso de coinfección VIH/VHC se encuentra en el sexo femenino, a diferencia de Paraguay donde la mayor prevalencia de los casos está en el sexo masculino $76 \%$ versus $24 \%$ para el sexo femenino 9 .

Con relación al tratamiento de personas coinfectadas con VIH/VHB es importante contar en su esquema antirretroviral para VIH con Tenofovir Disoproxil Fumarato (TDF) y Lamivudina (3TC) para lograr también la supresión viral del $\mathrm{VHB}^{10}$, también se puede contar con otros fármacos para el tratamiento de VHB como entecavir, emtricitabina, telbivudina o adefovir, sobre todo cuando no existe la coinfección ${ }^{2,8}$. No se aconseja utilizar como monoterapia Lamivudina con infección VHB y en coinfectados por $\mathrm{VHB} / \mathrm{VIH}$, debido a que este antirretroviral posee baja barrera genetica ${ }^{11}$. En el estudio, el $80 \%$ de los coinfectados cuenta con ambos fármacos dentro su esquema antirretroviral, a diferencia de otro estudio donde solo el 1,8\% contaba con esta coformulación ${ }^{8}$.

Resalta que el $12 \%$ de los casos con coinfección $\mathrm{VIH} / \mathrm{VHB}$ se encuentran en la población de $\mathrm{HSH}$, menor que la observada en Argentina $50 \%^{2}$ y España $59,3 \%{ }^{8}$. Es notable que existe un riesgo de coinfección $\mathrm{VIH} / \mathrm{VHB}$ de 5,5 veces más, en HSH a la población general.

No existieron casos de coinfección $\mathrm{VIH} / \mathrm{VHC}$ en $\mathrm{HSH}$, a diferencia de los casos encontrados en Argentina 11,2\% $\%^{2}$ y España $94 \%^{12}$. Puede ser atribuido por el reducido uso de drogas parenterales en Bolivia por el momento.

Ninguna de las personas con resultado positivo para VHB fueron vacunadas y de las 195 personas con $\mathrm{VIH}$, solo $18(9,2 \%)$ se encuentran inmunizadas contra este virus; por lo cual es imperativo que todos los pacientes diagnosticados con VIH y tamizados para VHB reciban la inmunización como prevención. Sin embargo, la respuesta a la vacuna frente al VHB en personas con VIH es menor que en los adultos no infectados ( $25-58 \%$ vs. $>90 \%$ respectivamente) ${ }^{13}$.

\section{CONCLUSIÓN}

Las seroprevalencias de la coinfección de hepatitis $\mathrm{B}$ o hepatitis $\mathrm{C}$ en las personas con $\mathrm{VIH}$ del Instituto para el Desarrollo Humano, son menores a las encontradas en estudios regionales.

Es imperativo ofertar las pruebas para hepatitis $\mathrm{B}$ y $\mathrm{C}$ a todas las personas con $\mathrm{VIH}$ para determinar medidas de salud pública y contar con datos epidemiológicos precisos; toda persona con $\mathrm{VIH}$ se debe realizar inmediatamente la inmunización para hepatitis $B$ y así prevenir la coinfección; haciendo énfasis en grupos vulnerables como los $\mathrm{HSH}$.

Es fundamental el abastecimiento de dosis para la inmunización completa contra el VHB en todos los servicios de salud público del país. Al momento se evidencio carencia en nuestro sistema de salud. En casos de encontrar la coinfección $\mathrm{VIH} / \mathrm{VHB}$ se debe adecuar el esquema de tratamiento antirretroviral para ambas patologías conteniendo Tenofovir y Lamivudina, para evitar complicaciones futuras.

También es importante realizar tamizaje permanente para $\mathrm{VHC}$ en personas con $\mathrm{VIH}$ para que las personas con esta coinfección tengan acceso al tratamiento para la hepatitis $C$, disponible en el país. 


\section{REFERENCIAS}

1. Soares Sampaio A, Arraes de Alencar L, Mendes Freire $P$, et al. Prevalencia de la co-infección con hepatitis B y C en pacientes HIV positivos y factores de riesgo asociados. Buenos Aires [Internet]. 2009 [citado en 2019 Agosto 20]; 17 (63): 12-17. Disponible en: https://www. huesped. org.ar/wp-content/uploads/2014/09/ASEI-63-12-17. $p d f$

2. Laufer N, Quarleri J, Bouzas M, et al. Coinfecciones por VHB y VHC en pacientes VIH positivos en la "era HAART": nuevos desafíos. MEDICINA (Buenos Aires) [Internet]. 2007 [citado en 2019 Agosto 20]; 67(1): 82-91. Disponible

en: http://www.medicinabuenosaires.com/revistas/ vo[67-07/1/completo/v67-1-p82-91.pdf

3. Toro A, Restrepo J. Hepatitis B. Medicina \& Laboratorio [Internet]. 2011 [citado 2020 Abr 20]; 17: 311-329. Disponible en: https:// www.medigraphic.com/pdfs/medla6/myl-2011/my[11786.pdf

4. OPS. La hepatitis B y C bajo la lupa. La respuesta de salud pública en la Región de las Américas [Internet]. 2016 [citado 2019 Ago 20]. Washington, D.C. Disponible en: https:/iris.paho. org/bitstream/handle/10665.2/31447/9789275319291spa.pdf? sequence $=5$ \& is A Alowed $=y \% 20$ )

5. Ramírez-Hita S. Problemas metodológicos en las investigaciones sobre VIH/SIDA en Bolivia. Ciência \& Saúde Coletiva [Internet]. 2013 [citado 2019 Ago 22]; 18(5):1321-1329. Disponible en: https://dxdoi.org/10.1590/S141381232013000500017

6. Padrón $A$, Reyes $A$, Hernández $V$, Jam $B$, Bouza Y, Cabanes $P$, et al. Coinfección VIH hepatitis B y C en la provincia de Cienfuegos. Rev Cubana Med Trop [Internet]. 2008 [citado 2019 Ago 22]; 60(2):141-7. Disponible en: http:// scielo. [ld.cu/scielo.php? script $=$ sci_arttexteIpid $=$ S037507602008000200007 \&'ing=es

7. Gutiérrez $C$, D'Angelo $P$, Sánchez $D$, Ameli G, Chacón E. Coinfección de hepatitis B y/o hepatitis $C$ en pacientes infectados por virus de inmunodeficiencia humana (VIH). Rev Soc Ven Microbiol [Internet]. 2012 [citado 2019 Ago 22]; 32:153-156. Disponible en: http:// ve.scielo.org/scielo.php? script $=$ sci_arttextoL $p i d=S 1315$ 25562012000200014 \& $\mathrm{I}$ ing $=e s$

8. Ruiz-Artacho $\mathrm{P}$, Téllez-Molina MJ, Vergas-García J, et al. Coinfección por VlH/ VHB: estrategias terapéuticas y control serológico. Rev Clin Esp [Internet]. 2013 [citado
2019 Ago 22]; 213(6):285-290. Disponible en: https://www.sciencedirect.com/science/article/abs/pii/ S0014256513000738?via\%3Dihu6

9. López G, Insfrán I. Coinfección VIH con Hepatitis B (Hvb) y Hepatitis C (Hvc) en el Laboratorio de Referencia del Programa Nacional De Control de VIH/SIDA. Rev. Inst Med Trop [Internet]. 2012 [citado 2019 Ago 22]; 7(1):19-26. Disponible en: https://www.msp6s.gov.py/dependencias/imt/uploads/Documento/v7n1a4.pdf

10. Huang $\mathrm{Y}-\mathrm{S}$, Chang $\mathrm{S}-\mathrm{Y}$, Sheng $\mathrm{W}-\mathrm{H}$, Sun $\mathrm{H}-\mathrm{Y}$, Lee $\mathrm{K}-\mathrm{Y}$, Chuang $\mathrm{Y}-\mathrm{C}$, et al. Virological Response to Tenofovir Disoproxil Fumarate in HIV-Positive Patients with LamivudineResistant Hepatitis B Virus Coinfection in an Area Hyperendemic for Hepatitis B Virus Infection. PLoS ONE [Internet]. 2016 [citado 2019 Ago 22]; 11(12). Disponible en: https://dx.doi. org/10.1371\%2Fjournal.pone.0169228

11. Buti M, García-Samaniego J, Prieto M, et al. Documento de consenso de la AEEH sobre el tratamiento de la infección por el virus de la hepatitis B (2012). Gastroenterol Hepatol [Internet]. 2012 [citado 2020 Abr 20]; 35(7): 512-528. Disponible en: http://aeeh.es/wp-content/ uploads/2012/09/Documento-de-consenso-VHFB-de-laAEEH.pdf

12. Lozano E, Rodríguez C, Ayerdi O, et al. Hepatitis C: nuevos diagnósticos y seroconversiones en una clínica de infecciones de transmisión sexual en Madrid. Rev Esp Quimioter [Internet]. 2019 [citado 2020 Abr 20]; 32(5): 440-444. Disponible en: https://www.nc6i.nlm.nif.gov/pmc/ articles/PMC6790886/pdf/revespquimioter-32-440.pdf

13. Fernández $M$, Rodríguez $O$, Brandy $A$, et al. Uso de vacuna frente a hepatitis $B$ adyuvada con AS04C en pacientes VIH. Rev Esp Quimioter [Internet]. 2018 [citado 2019 Ago 22]; 31(2): 105-109. Disponible en: https://www.nc6i. nlm.nif.gov/pmc/articles/PMC6159376/pdf/revespquimioter-31-105.pdf 